\title{
Healing leadership: the serving leader's impact on patient outcomes in a clinical environment
}

\author{
This article was published in the following Dove Press journal: \\ Journal of Healthcare Leadership \\ 27 April 2012 \\ Number of times this article has been viewed
}

\author{
Chris Nagel' \\ Anthony C Andenoro ${ }^{2}$ \\ 'Medical Operations - Continuous \\ Improvement, Cleveland Clinic, \\ Cleveland, OH, USA; ${ }^{2}$ Department of \\ Organizational Leadership, Gonzaga \\ University, Spokane, WA, USA
}

\begin{abstract}
The future of health care is a topic that has significant importance to patients and caregivers alike for generations to come. As the health care industry becomes more complex, leadership and the examination of how to most effectively apply it to meet efficiency standards and optimize the patient experience will become paramount. Through this paper the authors provide the foundation for meeting this need through an innovative and socially adept framework that identifies the critical character attributes of a serving leader and the powerful impact that serving leaders can have on patient outcomes in the health care setting. This framework is grounded in a leadership theoretical foundation and contextually examined through qualitative methods. As the business of health care becomes more complex and more competitive, finding ways to improve processes and create healing environments conducive to improved patient outcomes will differentiate average health care and excellent health care in the future. This paper provides the impetus for successfully addressing these needs through the development of serving leaders, and three specific characteristics: emotional care, presence, and awareness.
\end{abstract}

Keywords: servant leadership, health care, patient outcomes

\section{Introduction}

The challenge of delivering quality patient care consistently serves as a major discussion topic within today's demanding and complex health care landscape. Callahan ${ }^{1}$ writes that, today, health care is delivered in extraordinarily complex systems with extraordinary issues. Further, health care is more confusing and multifaceted than it has ever been. Health maintenance organizations, preferred provider organizations, and complex hospital bureaucracies have replaced the days when the physicians simply made house calls. Health care reform policies have placed new complexities on the industry, raising the need for improvements in care delivery. There is a transformation occurring in health care, and the accompanying efforts create the momentum for a revolution that could draw resemblance to the critical nature of the industrial and technological revolutions of the past. Seymour ${ }^{2}$ writes: "society appears to be sending a clear message to the nation's hospitals: take care of more people who have growing expectations and more complex medical needs while providing increasingly sophisticated care with relatively fewer resources." As such, discussions about leadership development methodologies in health care that shift leaders from a "command and control" environment to a "serving leader"-led culture will be vital as hospital staff attempt to meet the needs of the health care revolution.

Health care has made its way into both the national and international spotlight in recent years. Debate has consistently centered on how the United States' health 
care system can improve efficiency while producing better outcomes for the patient. Physicians who were influential in a "simpler" health care system in decades past have now seen the power switch to the government, insurance companies, and ultimately the patients. This trend forces the physicians to not only rely on their medical education but also develop business and leadership acumen. Kilpatrick ${ }^{3}$ notes that "leaders of many health care organizations have been defiant in serving both their employees and patients... many health care organizations and their leaders in the 20th century embraced an impersonal, technical, and financially focused approach to management."

The mentality that Kilpatrick speaks about must shift as health care becomes a commodity. Hospital leadership teams who look at their operation in order to deliver on a new model of health care and make these needed enhancements will be the organizations that outlast and outperform the others. The question at the core becomes what type of leader should provide the direction to take these institutions into the future, deliver high-quality outcomes and experiences for the patient, and balance the financial demands with the need for more efficient systems of health care.

Regardless of age, race, religion, sexual orientation, or nationality, everyone will be impacted by the health care system in his or her lifetime. Thus, the need exists for a modern system that establishes confidence, expresses compassion for the patients, and provides consistently high standards for patient care. This need is as vital to society as the need for nutritious food, clean water, and sufficient shelter. The United States struggles to keep pace with many other countries with respect to the developments in the fields of health care and patient satisfaction outcomes. Hence, the way that these hospitals practice leadership will determine the role they play in successful health care in future. Souba ${ }^{4}$ says that there is no other industry with more need for leadership development than that of the health care field. However, despite the ever-present debate over health care administration and its accompanying efficiency, one constant remains. Leadership will be key as facilities attempt to develop a framework that will care for the sick and maintain sustainability throughout a future rich with crisis and controversy.

Today's health care environment has created numerous touch points for patients through individuals not traditionally identified as specialty skilled professionals, such as physicians or nurses. For example, the housekeeper who cleans the room may have as many interactions with patients and family members as the nurse or physician does. Hence, the need for serving leaders at all levels of the organization is critically important. There is an influx of professionals and frontline staff from other industries resulting from downsizing in other sectors of the economy. Therefore, leadership in health care organizations in the future will not base leadership on specialty skill background alone but on a focused balance with innate leadership competencies. These serving leaders, as noted in the philosophy of servant leadership ${ }^{5}$ will help others grow and create other serving leaders in order to sustain the impact on patient outcomes from the senior level leader to the frontline caregiving leaders.

There is minimal empirical research on the overall effectiveness of being a serving leader and its impact on employee engagement and patient satisfaction ${ }^{6,7}$ although quantitative research has traditionally grounded implementation and change management efforts. We are entering a new era of health care that will call for new paradigms of understanding how to empower leadership. This paper challenges the outdated idea that the effect of leadership stops at patient satisfaction and employee engagement, and examines the impact of being a serving leader on patient outcomes. This research is critical to the complete health care system, from the frontline caregivers to the hospital leadership, insurance providers, or government payers of our nation's hospitals. These improved outcomes benefit the people whom the health care community aims to serve the most: the patients and families suffering from the diseases afflicting populations today. This type of research will be critical as we continue to develop and evaluate new technologies and consider the human piece within the healing puzzle. The following proposes a framework for the creation of a new generation of serving leaders with the ability to guide the way to a healing leadership model of care and to revolutionize health care for the future.

\section{Leadership traits for health care's future}

A search for characteristics of a serving leader will return an abundance of information, all of which has merit in discussions about the future of health care and leadership. Of these, there are a critical few themes revealing themselves as consistent around which would be critical to enhance the experience of a patient in the health care environment. The essence of the serving leader revolves around a state of being and traits consistent with the notion of holistic care or treating the whole person including the mind, body, and spirit rather than simply the body or disease.

MacLeod and McPherson ${ }^{8}$ define a person in the context of the health care setting using Cassell's "simplified 
description of a person." They say that people should not be treated like an "it." It is equally important to view the patient as a legitimate other in the process of health care delivery by recognizing the differences and stories that each person represents. They continue that "in this tradition, healing comes from within the person, clearly contrasting with modern medical care with its emphasis on evidence-based practice, protocols, technological advances, and the right way of dealing with things."

In the vast array of characteristics, it would be difficult to narrow the field to a few distinct relationships that create a healing environment for the patients and families leading to certain positive physiological reactions. Within the serving leader framework, several traits are identifiable as supportive of the patient care process. However, three traits consistently emerge as themes that address the ability to garner a state of being, go beyond the behavioral domain, and create a foundation for practice grounded in attitudes and dispositions: emotional care, presence, and awareness. The Schwartz Center ${ }^{9}$ conducted a nationwide survey of 800 patients and 500 physicians on the impact these have on patient experience and, ultimately, outcomes. The center found that an overwhelming majority of the responders felt that "health care delivered with compassion (including presence and awareness factors) can make a difference in how well a patient recovers from illness - it can also make a difference in whether a patient lives or dies."

This powerful idea has huge implications as health care organizations move to find ways to enhance the patient experience to include outcomes. These ideas affirm the discussion that each caregiver, regardless of their role in the organization, has the ability to be part of the healing process for the patient and family.

\section{Emotional care}

Emotions can be a complex system of interactions that involve the physiological reactions of a person related to influence placed on them from factors both internally and externally. ${ }^{10}$ Humans, in stress-filled situations like a hospital, have numerous emotional reactions with wide-ranging forces. Many times the patients and families enter the system stressed, based on fear and anxiety by the unknown of what is happening. The need to transition them from a negative emotional state to a positive one becomes critical in structuring an environment for healing and the holistic philosophy. ${ }^{11}$

Emotional care in health care develops out of a conceptual framework including compassion, empathy, love, and hope. These characteristics are, by the very nature of the work in the hospital environment, critical to the patient experience and can potentially affect outcomes with the creation of a healing environment. Inherent in the health care setting is the pain and suffering of not only the patient but also the family. Pain and suffering are constant themes in the hallways of hospitals, so emotional caring and the related characteristics can serve as the potential antidote to ease the discomfort patients and families feel. This is not to imply that pain and suffering will go away completely, but leaders who possess and are able to utilize these characteristics with their patients could assist in the transition from a declined emotional state to a positive emotional state, which supplements the healing process.

Van der Cingel $^{12}$ states that an emotion from a person always has an object connected to it. Most who have experienced the health care system understand the scary proposition presented by various terminology, instruments, technology, and the fear of the unknown. The actions of caregivers involved in these emotional components for the patients and families can assist in improving mental state, ultimately leading to less anxiety. ${ }^{13}$ This decrease in anxiety is at the heart of the holistic healing model of care. Additionally, it has the ability to lead to a more conducive environment for healing and improved outcomes.

The conundrum is that today's health care system is more challenging and demanding than ever. The added pressure of a reduced work force has led to a reduction of caring behaviors in the health care community. Evidence has shown that caregiver compassion has fallen and, with the extra workload, caregivers are experiencing a new phenomenon called compassion fatigue. A Schwartz Center study determined that there is a critical need for more compassion in the hospital and that there is a real belief that today's health care and the involvement of the emotions of a patient are important. However, the struggle is making that a reality in the "business" of health care. This gap, highlighted in a study where $83 \%$ of the patients said that they expect the physicians to treat them with compassionate care, but only $67 \%$ of those same patients indicate that they received that kind of care, tells the story of our current environment. ${ }^{9}$ Emotions will always be a part of the human DNA, but it is the duty of the hospital leadership to ensure that the patients and families feel that compassion, empathy, and hope that make up the key emotional drivers related to the emotional care of patients by being a serving leader.

\section{Presence}

Presence not only relates to the health care environment but to society as a whole. Today's society, consistently drawn in 
several directions and held to quotas and standards while being inundated by technologies and information overload, is struggling to hold on to personal interactions. Technologies that are supposed to make life easier are competing for our attention, such as social media, pagers, iPods, BlackBerry devices, and tablet computers, for example. These items have brought health care professionals to a society that reestablishes what it means to interact with others and devalues depth of relationships that can lead to a better understanding of the patient and his or her needs. Hu notes that society will become isolated and lose all touch with real human interaction, and further asserts that individuals would rather send a text message or email than have a real face-to-face conversation. ${ }^{14}$ This trend is at the heart of the discussion around the need for presence in the hospital setting. Our society has lost the art of personal interactions, and simply having a conversation with another person is becoming a burden and presents considerable challenges for the next generation of caregivers.

Presence comes from the Latin word "praesentia", meaning to be with someone. ${ }^{15}$ However, Senge ${ }^{12}$ challenges the current thinking associated with this term to provide more meaning. He writes that society has traditionally thought of the idea of presence to be attentive of the environment you are in. However, current thinking suggests that there is a need to "appreciate presence as deep listening and being open beyond one's preconceptions and historical ways of making sense. This opens up thinking and creates a future of limitless possibilities, including potential impact for patients.

These trends do not stay on the exterior of our nation's hospitals. They have an effect on interactions that occur within the walls of the patient rooms on a daily basis. The value of time as our workforce surges to the limits of productivity and time becomes split between answering emails, texts, and pages that, in turn, limit one's ability to connect on a personal level with the patients. The boardrooms, hallways, and meeting rooms show a trend where there are more people answering phones, emails, and pages in the middle of discussions with another person, even patients. Presence, in this context, refers to putting those distractions aside and genuinely being with the other person, which in this case means the patients and their families.

Presence does not refer to just being in the same physical location of the person, but rather engaging in real dialog to gain deeper mutual understanding. Kline ${ }^{16}$ outlines several components of this type of dialog. She identifies attention including eye contact, equality, appreciation, and place as just a few of the ways to enhance the environment and truly become intimate with the patients and families.

Gordon ${ }^{17}$ highlights that presence is not simply the idea of listening to another person or being. It is much more than that. When professionals are present with a person, they demonstrate the willingness to create an environment grounded in the I-you dialog where the other person is not an object but rather a person who has feelings and emotions. The absence of this kind of dialog creates a situation where the person is an object or an "it" whom that professional is talking to rather than with. This is referred to as the I-it dialog. The I-you dialog can exist if a professional becomes truly present with the other person and desires to serve and coexist with them. The days when the caregiver is the expert and is charged with determining the patient's best course of action are diminishing and being replaced with a more collaborative idea of patient-centered care where the patient is a critical part of their care process. Presence is the foundation for reciprocity and this mutually beneficial relationship.

\section{Awareness}

Third River Partners ${ }^{18}$ highlight awareness as a vivid representation of what makes up personality to include a multifaceted array of items such as strengths, weaknesses, values, and beliefs, for example. The author goes on to highlight that having self-awareness allows individuals to recognize needed change and react to that change in an appropriate way in order to move toward being with another.

Awareness begins with the self and is the essence of being a serving leader. ${ }^{5}$ The father of the servant leadership movement, states:

The servant-leader is servant first... It begins with the natural feeling that one wants to serve, to serve first. Then conscious choice brings one to aspire to lead. That person is sharply different from one who is leader first, perhaps because of the need to assuage an unusual power drive or to acquire material possessions...

The health care field has traditionally been a command and control culture. The shift has begun and the patients are becoming more educated and knowledgeable about their health care options. As such, it is necessary for these caregivers to be aware of their role as servant first and fostering the ability of them to meet the needs of the patient and family in a much different way.

This does not come without great pain on the part of the caregiver, as humility must be a critical component of the awareness discussion. It is difficult for anyone to look 
critically at oneself, and even harder to initiate the change. However, to truly change health care and affect the outcomes with these behaviors, self-awareness and humility must be present within the leadership identity of an individual. Coulehan ${ }^{19}$ adds: "the new professionalism movement in medical education takes seriously the old medical virtues. Perhaps the most difficult virtue to understand and practice is humility, which seems out of place in a medical culture characterized by arrogance, assertiveness, and a sense of entitlement."

Herein lies one of the issues of changing the health care culture to one with a serving heart. It must begin with awareness and humility before accessing the behaviors that are needed to change the face of health care for future generations.

\section{Health care leadership: a new day}

A patient or family member, simply by the very nature of their need to enter into the health care system, experiences stress and anxiety. The patient's overwhelming stress and heightened sense of anxiety can have a detrimental effect on the body and mind and create additional challenges for professional staff attempting to assess the patient's health and projected outcomes. This combination has the potential, according to DeLongis et al, ${ }^{20}$ to lead to additional illness and the inability to use the mind as a useful tool in the healing process. Fear of the unknown, one's own mortality, anxiety, depression, and pain are only a few of these influences that can come over the individuals and affect their care. In fact, there is some evidence that "illness worry" can lead to a reduced ability to recover or heal, resulting in an increase in health care costs in the nation. ${ }^{11}$

The discussion of this holistic approach to health care is grounded in how the World Health Organization ${ }^{21}$ defines holistic health. It provides that:

... viewing man in his totality within a wide ecological spectrum, and emphasizing the view that ill health or disease is brought about by an imbalance, or disequilibrium, of man in his total ecological system and not only by the causative agent and pathogenic evolution.

This identifies that the healing process lies within the mental state of the person, as much as it does with the disease and accompanying treatment plan. The factors and behaviors of emotions, presence, and awareness provide considerable support for creating this holistic approach.

This conclusion creates a case for continued dialog relating to the impact of serving leadership on patient outcomes and the increased priority for treating the whole person.
The mind has a strong impact on many aspects of our world, and how individuals view experiences and react emotionally will create a positive or negative result. In the case of hospitals and health care units, it is increasingly important to keep the mind filled with positive energy. This positive energy can create a positive result in staying healthy and ultimately improving outcomes for the patient.

\section{Impact of healing leadership}

Although the discussions are strong about the need to treat the whole person to include the mind, spirit, and body, there is very little evidence to make the claim that behaviors of our health care workers including leadership revolving around such things as emotions, awareness, and presence will have any effect on the outcomes for the patients. However, some evidence does exist that shows that serving leader behaviors have an impact on other groups led by leaders practicing these behaviors. Southwest Airlines is a great example of how espousing serving leader principles can make a difference. In the competitive world of air travel, Southwest has achieved 37 consecutive years of profitability, ranked number one in customer satisfaction in 2009 by the US Department of Transportation, and named by Fortune magazine as one of the world's most respected companies 13 years in a row, including recognition as one of America's top workplaces. Southwest Airlines has grounded its practice in the idea that people are more than numbers or dollars, and has empowered its employees to serve with compassion and empathy. Although this company operates outside of the current health care mindset, the results of this practice are transferable and provide an excellent example of how to positively influence the customer experience.

There are health care organizations taking on this cultural transformation through a serving leadership lens that understand its implications for the future of health care. An example of this is the work the Cleveland Clinic has embarked on. The Cleveland Clinic has collaborated with Third River Partners to begin this journey with over 50 of its leaders certified in the Serving Leader Toolkit based on the book, The Serving Leader, by Ken Jennings and John Stahl-Wert, ${ }^{22}$ using five powerful actions. This certification process, facilitated by Amy Foster from Third River Partners, delivers an actionoriented experience allowing participants the opportunity for learning and practical opportunities to integrate the learning in real situations. Coupled with situational and personal coaching, this cohort learning approach has created a groundswell of excitement, creating a pull for additional sessions around the organization. In a postcertification, after-action 
review, $100 \%$ of the respondents indicated that they experienced personal growth and $64 \%$ experienced a major personal impact. Subsequently, $83 \%$ shared documented examples of how improved leadership styles enhanced collaboration with teams, productivity, and results. ${ }^{23}$

Stuckey and Nobel ${ }^{21}$ concluded that less anxiety and stress can have a positive effect on the immune system and its functionality specifically related to pain in cancer patients. The challenge is that there are no current research efforts addressing the combination of the serving leadership behaviors with a correlation to creating an environment that can improve patient outcomes. There is a need for more research similar to the Cleveland Clinic to begin to move this theoretical framework into grounded research.

\section{Confirmability through qualitative inquiry}

Due to the general lack of empirical grounding for this idea in the literature, the authors felt compelled to informally assess various health care facilities and those that work within them in an effort to examine confirmability. ${ }^{24}$ The authors qualitatively explored three contexts (large trauma one hospital, small regional hospital, and an urgent care facility) and conducted unobtrusive site visits. ${ }^{25}$ These ultimately served as an opportunity to view the richness of the context and gain perspectives through observation and informal interactions with individuals within the contexts. Data were analyzed via a constant comparative analysis, ${ }^{26}$ and themes emerged naturalistically. These visits yielded complementary data to what was found in the literature. Specifically, two consistent themes emerged. First, when patients and family were treated with respect, smiles, and compassionate behaviors (eg, social awareness, empathy, and consideration), they responded in similar manners. These interactions seemed to have a calming effect on each of the patients within the contexts and actually led to deeper conversation between the patient and their families, which were grounded in supportive language. Second, and conversely, when the patients were treated impersonally and without respect to their individuality, the attending staff met with resistance from the patients and increased levels of anxiety. The more powerful finding was that the families also had a more difficult time demonstrating supportive behaviors for the patient when compassion was not presented from attending staff. Often, the families would just sit in silence or focus on external stimuli (eg, the waiting room television or magazines) when the attending staff lacked compassion.
This leads the authors to assert that more research is needed to fully examine the contexts and resulting implications of the serving leader. However, it is evident that patients benefit from compassionate interactions with attending staff that are grounded in serving leader behaviors, emotional care, presence, and awareness.

\section{Conclusions and recommendations}

Discussion around how the health care community transitions into a new age of leadership is happening, and serving leadership is at the forefront of the conversation. The literature indicates similarities between the characteristics of the "serving leader" and what patients believe is critically important. This was also confirmed by emergent themes stemming from the qualitative data collection efforts. Thus, the future of the health care industry will be grounded in the evidence that these traits are critical for, and should be expected in, health care environments. Emphasis in years past has been on the technical skills of the caregiver. However, now more than ever, health care administrators need to recognize that the development of leadership competencies with individuals at all levels of the health care facility will provide a critical partner for the technical skills in an effort to meet the needs of the patients that these facilities serve.

The idea is that serving leaders can positively influence patient outcomes, and there is a different leadership model needed in our hospitals today incorporating healing leadership competencies. The study and development of these competencies in our health care leaders, coupled with the already existing technical skills, could be the future of health care.

These factors make up a cycle for patient care that provides an ultimate experience for the patient, including the treatment of the mind and body necessary for holistic care. If one of these factors is not present, the treatment and care of a patient cannot be optimized and will inevitably lead to less positive patient outcomes. It is not enough to treat the causation and the disease. Health care administrators have a moral responsibility to treat the mind and spirit of those whom they serve.

The challenge the industry faces is that currently no data exist to support a claim that the components of emotional care, presence, and awareness can lead to better outcomes for patients. More qualitative research is needed in order to determine the impact that healing leadership can have on the health care industry and its constituents. The evidence we do have is informal and largely anecdotal, and the traditional health care industry will demand data and hard facts in order 
to move in this direction. However, this idea provides health care with direction, and that is something that is sorely needed in this complicated landscape that supplants traditional health care practices.

The health care industry has reached a point where discernment must lead to action. That action can be grounded in the desire to practice healing through leadership. The difference in tomorrow's health care landscape will be the ability of caregivers to complement their technical skills with emotional care for the patient, genuine presence, and full awareness of the opportunity to create the change that is needed to serve those who need it most.

\section{Disclosure}

The authors report no conflicts of interest in this work.

\section{References}

1. Callahan C. Leadership and healthcare perfection. US Army Medical Department Journal. 2009:5-10.

2. Stefl M. Common competencies for all healthcare managers: the healthcare leadership alliance model. J Healthc Manag. 2008; 53(1):360-374.

3. Kilpatrick AO. The healthcare leader as humanist. $J$ Health Hum Serv Adm. 2009:451-465.

4. Barger J. Step back from the baggage claim: healthcare leader edition. Columbus, OH: One Love Publishers; 2010.

5. Definition of Servant Leadership. (2011). Retrieved from http://www. greenleaf.org/whatissl/. Accessed April 03, 2012.

6. MacLeod D, Clarke N. Leadership and employee engagement: passing fad is a new way of doing business. The International Journal of Leadership in Public Service. 2010;6(4):26-30.

7. Babcock-Roberson ME, Strickland O. The relationship between charismatic leadership, work engagement, and organizational citizenship behaviors. J Psychol. 2010;144(3):313-326.

8. MacLeod R, McPherson K. Care and compassion: part of person-centered rehabilitation, inappropriate response or a forgotten art? Disabil Rehabil. 2007;29(20-21):1589-1595.
9. Young K. Survey: compassionate care could mean life or death. Medical Ethics Advisor. 2011;27(1):1-12.

10. Papathanassoglou E. Psychological support and outcomes for ICU patients. Nursing in Critical Care. 2010;15(3):118-128.

11. Christensen KS. The outcome if health anxiety in primary care. A twoyear follow-up study on health care costs and self-rated health. PLoS ONE. 2010;5(3):e9873.

12. Van der Cingel M. Compassion and professional care: exploring the domain. Nursing Philosophy. 2009;10(1):124-136.

13. Yu D, Lee D, Woo J. improving health-related quality of life of patients with chronic heart failure: effects of relaxation therapy. $J A d v$ Nurs. 2010;66(2):392-402.

14. Hu T. A hurting society. World Future View. 2010:42-44.

15. Definition of presence. Available from: http://dictionary.reference.com/ browse/presence. Accessed March 15, 2011.

16. Kline N. A Time to Think. London: Ward Lock; 1999.

17. Gordon M. Listening as embracing the other: Martin Buber's philosophy of dialogue. Educational Theory. 2011;61(2):207-219.

18. Jennings K. The Serving Leader Toolkit. Third River Partners, LLC; RTGP 12; 2010.

19. Coulehan J. On humility. Ann Intern Med. 2010;153:200-201.

20. DeLongis A, Folkman S, Lazarus RS. The impact of daily stress on health and mood: psychological and social resources as mediators. J Pers Soc Psychol. 1988;54(3):486-495.

21. Stuckey HL, Nobel J. The connection between art, healing, and the public health: a review of current literature. Am J Public Health. 2010;100(2):254-263.

22. Jennings K. Stahl-Wert, J. The Serving Leader. San Francisco: BerrettKoehler Publishing; 2004.

23. Hyde H, Jennings K. The Greater Goal: Connecting Purpose and Performance. San Francisco, CA: Berrett-Koehler Publishers; 2012.

24. Erlandson DA, Harris EL, Skipper BL, Allen SD. Doing naturalistic inquiry: a guide to methods. Newbury Park, CA: Sage Publications, Inc; 1993.

25. Denzin NK, Lincoln YS. The SAGE handbook of qualitative research. Thousand Oaks, CA: Sage Publications, Inc; 2011.

26. Glaser BG, Strauss AL. The discovery of grounded theory. Hawthorne, NY: Aldine; 1967
Journal of Healthcare Leadership

\section{Publish your work in this journal}

The Journal of Healthcare Leadership is an international, peer-reviewed, open access journal focusing on leadership for the health profession. The journal is committed to the rapid publication of research focusing on but not limited to: Healthcare policy and law; Theoretical and practical aspects healthcare delivery; Interactions between healthcare and society and evidence-based practices;

\section{Dovepress}

Interdisciplinary decision-making; Philosophical and ethical issues; Hazard management; Research and opinion for health leadership; Leadership assessment. The manuscript management system is completely online and includes a very quick and fair peer-review system. Visit http://www.dovepress.com/ testimonials.php to read real quotes from published authors. 